family, with the additional problems at home arising from the excessive washing involved. It is no comfort to child or parent to be told, "He will grow out of it."

We feel, then, that it is unfair to dismiss a potentially useful treatment so sweepingly. Surely it would be more sensible for us to aim to educate parents to treat the drugs with caution, and to make more use of childproof containers both for syrup and for tablets.

SusAN D Dickson

Enuresis Clinic,

Doncaster Royal Infirmary,

P DICKSON

Hatfield,

Doncaster, Yorks DN7 6JX

SIR,-Against the growing sophistication of modern educational institutions the effects of persistent bed-wetting in terms of the emotional and social development of enuretic sufferers are not always "benign," as your leading article (17 March, p 706) states. May I therefore suggest an approach which can readily be used by clinical medical officers in enuresis clinics operating from school health premises ?

In cases of persistent enuresis cystometric examination of bladder function indicates that in the instances examined bed-wetting is the consequence of a developmental disorder of the bladder musculature. ${ }^{1}$ If therefore the tricyclic antidepressants imipramine and amitryptiline are used for their direct action on smooth muscle ${ }^{2}$ success rates of above $90 \%$ can be obtained. In this context the timing of administration is more important than the actual dosage; and by combining chemotherapy with bladder exercises, such as repeated bladder distension and voluntary retention of urine, the relapse rate can be reduced to acceptable proportions.

In general, the greater the age of the child the better the outlook, because in the older child his or her active co-operation can more readily be obtained.

GWYNNE V LEWIS

$$
\begin{aligned}
& \text { Health Department, } \\
& \text { Ipswich, Suffolk } \\
& \\
& { }^{1} \text { Bakwin, H, and Bakwin, R M, Behaviour Disorders in } \\
& \text { Children, p 442. London, W B Saunders Company, } \\
& 1972 . \text { S and De Quenedo, A, Disease. American } \\
& \text { Epstein, S J, and De } \\
& \text { fournal of Psychiatry, 1964, 120, 908. }
\end{aligned}
$$

\section{Malaria in Wolverhampton}

SIR,-We were very interested in the communications from Dr Eric Walker (16 December, $p$ 1718) and Dr C J Ellis and others (10 February, p 385) and would like to add our experience.

For the year ending 31 December 1978, we have diagnosed 158 new cases of malaria within the area of our health authority, compared with 112 cases in 1977 - a $42 \%$ increase, reflecting the present upsurge of this infection in the tropics. There have been no deaths from this infection. One hundred and twenty-six $(80 \%)$ cases presented between May and September. Seventy-six ( $48 \%$ ) cases were new immigrants. All but one case were from the Indian subcontinent and infected with Plasmodium vivax. The exception was a 30-year-old African male from Malawi who was infected with $P$ ovale. Three $(2 \%)$ Asians making a holiday visit to this country were also infected with $P$ vivax. The other $79(50 \%)$ cases were residents of this country who had been travelling abroad. One man who was infected with $P$ falciparum was a British worker returning from Nigeria. The remainder, most of whom were infected with $P$ vivax, were visitors to the Indian subcontinent, mainly the Punjab. However, there were two adults infected with $P$ falciparum (one of whom had a mixed infection with $P$ vivax) and three cases of $P$ malariae infection (one was mixed with $P$ vivax). Interestingly, about one-quarter $(19 / 78)$ of the travellers to the Indian subcontinent were children under 14 years of age. Those who were born in the UK may be more vulnerable to the dangers complicating infection with $P$ falciparum.

These figures of course represent only cases confirmed by examination of stained blood films in our laboratory and thus are baselines. It may be that there are many more cases which are diagnosed and treated clinically but which are not notified.

Our experience and that of others clearly demonstrates that the problem is increasing; it could be considerably reduced if much more attention were focused on informing travellers about this disease and its possible danger and educating them about its prevention. Publicity could be effectively promoted by campaigns carried out by the advertising media, with appropriate medical advice to ensure accuracy and relevance of information Such schemes, at least during peak travelling months, should include television advertisements, press articles, and talks on local radio stations, particularly if they are reinforced in the local dominant Asian language as well as English. General advice and prescriptions for prophylactic drugs are available from genera practitioners. However, prophylactic drugs are not available at the Health Service's expense, and so it should be publicised that they are cheap. Should these drugs be made available under prescribing regulations? It is most important to emphasise too that drugs must be taken continuously during the period abroad and for a period of at least a month after return to this country.

Such positive measures should bring to the overstretched resources of the NHS the additional reward of conserving valuable clinical time and services.

We thank our colleagues in the Malaria Reference Laboratory for their invaluable help.

Public Health Laboratory, ( Wolverhampton WV10 0QP

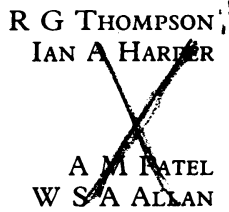

Haematology Laboratories,
Wolverhampton Area Health Authority

Liver injury, drugs, and popular poisons

SIR,-I was very interested to see the leading article on the wide range of drugs that affect the liver ( 3 March, p 574) but a little disappointed to read the following: "and after years of controversy halothane has now earned a definite place in the list of drugs causing liver disease." As an anaesthetist who greatly values halothane, I do not eliminate the possibility that it may (very rarely) adversely affect the liver, but I wish that hepatologists and others would be a little more open minded.

The implication of that statement is that there is a clearly proved case against the drug, and no longer any controversy. Reference to a paper ${ }^{1}$ by Sherlock is made in support of the statement. The paper in question, however, was included in the Lancet under the genera heading "Point of view." Surely one person's opinion, even an opinion as eminent as Pro- fessor Sherlock's, cannot be taken as the basis for such a dogmatic statement.

The article goes on to review the many proprietary preparations available that may affect the liver. One wonders how many of the patients labelled as having halothane hepatitis have had the possibility of ingestion of such agents eliminated from their histories.

\section{J A W WILDSMITH}

Department of Anaesthetics,

Royal Infirmary,

' Sherlock, S, Lancet, 1978, 2, 364

\section{Prescription for a better British diet}

SIR,-Dr $\mathbf{R}$ Passmore and others prescribe "for a better British diet" (24 February, p 527) and undoubtedly they succeed, but it is not the best. Though they base their prescription on present food supplies in the UK, they should have heeded the dietary goals prepared by the McGovern Committee for the US ${ }^{1}$ which are generally applicable to the UK.

Passmore et al make no recommendation about the use of high-extraction flour or of polyunsaturated fats. They state that bread "is rich in many nutrients"; but $87 \%$ of the bread being eaten is white, ${ }^{2}$ to which this statement hardly applies in comparison with bread made from high-extraction flour (which also contains fibre). The laudable reduction in meat will reduce saturated fat, but the overall reduction of $15 \%$ in total fat will not increase essential fatty acids (EFA, or certain polyunsaturated fatty acids). This increase is wisely recommended by McGovern. Dr J I Mann (17 March, p 732) follows McGovern in suggesting that the ratio of saturated to polyunsaturated fatty acids should be unity, but I join with Sir John McMichael (20 January, $p$ 173) against Mann in disliking transpolyunsaturated fatty acids (which are not EFA and tend to behave biologically as (saturated). Mann's statement that "diets rich in saturated fat are invariably high in refined carbohydrate and total energy intake" is not correct. Eskimos and mutton-eating Welsh in Patagonia had diets high in saturated fat with no refined carbohydrate; and so had Stefansson (who subsisted when I knew him mainly on fatty meat and red wine) and McCarrison (who ate meat and whole-grain bread with quantities of butter but very little sugar), and neither was obese.

The ratio of EFA to certain non-EFA (mainly saturated and trans-polyunsaturated fatty acids) is the relevant consideration, rather than total fat or total saturated fat. Eskimos, when on their traditional diet, had the highest fat intake in the world and no dietary fibre, but even relatively long-lived ones had almost none of our non-infective "Western" diseases (ischaemic heart disease and other thrombotic disorders, cancer, diabetes mellitus, dental caries, multiple sclerosis, intestinal disorders, varicose veins). But they had a diet very high in EFA from fish and seal, and rapid intestinal transit times. Passmore leaves fish unchanged, and unfortunately McGovern states that the fat of fish should be reduced in the diet to help reduce total fat; whereas I would greatly increase it-especially our most caught fish, mackerel, which is readily available and cheap (about 35 p a pound), and contains more polyunsaturated than saturated fatty acids as well as some other nutrients, yet only a small pro- 
portion of what we catch goes to the home market.

Obviously we need much more research, but enough is known to prescribe the best British diet from available knowledge.

Hugh Sinclair

International Institute of

Human Nutrition

Sutton Courtenay, Oxon OX14 4AW

US Senate Select Committee on Nutrition and Human Needs, Dietary Goals for the United States, 2nd edn. Washington DC, US Governmen Printing Office, 1977

Ministry of Agriculture, Fisheries and Food, House hold Food Consumption and Expenditure: 1977

SIR,-Your correspondent Dr Margaret Barker (10 March, p 681) raises a point which has intrigued me for a long time. Am I correct in assuming that ground-up wheat gives wholewheat flour? If so, I presume that white flour is produced by picking out the brown bits (and what do they do with these anyway-an EEC brown bit mountain?). In which case, it should be dearer than brown flour. Since this is not so, do they pick out the brown bits to make white flour and then add them back to make brown flour again, thereby increasing the price?

I have also wondered whether some brown bread really does contain the necessary brown bits or whether some of it is not just ordinary brown white bread-and how can we tell?

\section{HOWARD G HANLEY}

London W1N 1DL

\section{Labetalol and urinary metadrenalines}

SIR,-Interference by the antihypertensive drug labetalol in the estimation of urinary catecholamines and their metabolites has been the subject of recent correspondence ${ }^{1-3}$ and a report $^{4}$ in this journal. Spuriously raised urinary values were evident ${ }^{2-4}$ after treatmen both with a fluorimetric catecholamine assay ${ }^{5}$ and the standard spectrophotometric procedure $^{i}$ for total (free plus conjugated) metadrenalines (metadrenaline and normetadrenaline).

Because our own modification ${ }^{8}$ of this spectrophotometric procedure gives substantially lower blank values than the present method, we were prompted to investigate whether the problem could be overcome with its aid. We compared tota metadrenalines, as measured by our own modified Pisano technique, ${ }^{8}$ with values obtained when tota normetadrenaline (NMA) plus total metadrenaline (MA) were determined by a highly specific and sensitive gas chromatographic procedure. ${ }^{9}$ Most of the specimens we examined were kindly supplied by Drs $\mathrm{H} \mathrm{J}$ Dargie and $\mathrm{C} \mathrm{A}$ Hamilton and were from the same patients on whom they had already reported. ${ }^{4}$

We agree with others ${ }^{2-4}$ that labetalol gives rise to factitiously raised values for urinary total metadrenalines when the classical Pisano method is employed. However, values yielded by our modified procedure ${ }^{8}$ correlated well with thos obtained by gas chromatography, except in one patient out of eight studied. This patient had much the highest value of all when the original Pisano method was used, indicating that interference was great. Even here, however, the result obtained by our modified procedure was not markedly increased, although some interfering substance was obviously still present. It was found that maximum interference was not associated with maximum dose of labetolol.

Our simple improvement to Pisano's method, which involves three additional steps a pH adjustment of the column eluate, extraction into toluene, and back-extraction into potassium carbonate, seems to eliminate the interfering material in most cases. It has the advantage that it can be carried out where access to more specific gas chromatographic or radioenzymatic methods is not available. We therefore suggest its use for screening patients who are taking labetalol when a possible phaeochromocytoma is suspected. If occasional raised values are found, the sample might then be subjected to more careful scrutiny by one of the more specific procedures.

We are grateful to $\mathrm{Mr} \mathrm{M} \mathrm{W}$ Weg for helpful discussions.

Pauline Lax

C R J RUTHVEN MERTON SANDLER

Bernhard Raron Memorial Research

Laboratories and Institute of

Obstetrics and

Queen Charlotte's Maternity Hospital,

London W6 0XG

Harris, D, and Richards, D A, British Medical fournal, $1977,4,1673$.

Chapman, B P, Veitch, A G, and Shepherd, B,

British Medical fournal, 1978, 1, 364.

Kolloch, R, Miano, L, and de Quattro, V, British Medical fournal, 1979, 1, 268.

Hamilton, C A, et al, British Medical fournal, 1978, 2 800

'Crout, J R, Standard Methods of Clinical Chemistry, d D Seligson, vol 3, p 62. New York, Academi Press, 1961.

Euler, V S, and Lishaiko, F, Acta Physiologica Scandinavica, 1961, 51, 348 .

'Pisano, J J, Clinica Chimica Acta, 1960, 5, 406.
" Ruthven, C R J, and Sandler, M, Clinica Chimica Acta 1965, 12, 318 . Nelson, L M, et al, Clinica Chimica Acta, 1979, 92,
235.

\section{Difficulties in diagnosing meningococcal} meningitis

SIR, - We read with great interest the findings of Drs Oliver C Smales and Nicholas Rutter in their review of bacterial meningitis in children (3 March, p 588). The difficulties encountered in the initial clinical evaluation of bacterial meningitis are well appreciated, especially in the setting of prior treatment with antibiotics.

In recent years the use of the counterimmunoelectrophoresis assay has been helpful in identifying some of the more common pathogens seen in the setting of meningitis ${ }^{1}$. however, it is limited in terms of available antibody preparations, requires skilled technicians and strict quality control, and is not readily available in small hospitals.

The limulus endotoxin assay has proved to be a rapid (60-minute), reliable method in the initial evaluation of meningitis. On the basis of the findings of Levin and Bang ${ }^{2}$ that minute amounts of endotoxin released by Gram-negative bacteria produced gelation of amoebocytes from Limulus polyphemus (the horseshoe crab), multiple studies have since confirmed the usefulness of the limulus assay as an aid in the diagnosis of Gram-negative meningitis. ${ }^{3-5}$ In comparing the sensitivities of limulus assays and Gram-stain smears on initial spinal fluid samples of patients with Gram-negative meningitis who did not receive prior antibiotic therapy, positive limulus assays were obtained in at least $97^{\circ} \%$ of cases, whereas only $70^{\circ}$ had organisms visible on smears. ${ }^{3-5}$ Moreover, when the results of the limulus assay were compared with the results of Gram stain and culture after 24-48 hours of antibiotic therapy in 25 cases of culture-proved meningitis with Haemophilus influenzae or Neisseriameningitidis, Gram-stains were positive in $4(16 \%)$, cultures in $6\left(24^{\circ}{ }_{0}\right)$, and the limulus assay in $17\left(68^{\circ}{ }_{0}\right) .^{4}$ By contrast with the complexities involved with the counterimmunoelectrophoresis assay, the limulus assay could be used as a "bedside" test by house staff without sophisticated training."

The chief drawback of the limulus assay is that a negative test does not rule out the possibility of meningitis due to organisms other than Gram-negative bacteria. However, with a thorough understanding of the advantages and limitations of the limulus assay it can serve as a valuable adjunct to other diagnostic modalities in the initial clinical management of meningitis.

We none the less strictly agree with the authors that a strong index of suspicion, aggressive repeated evaluation, and appropriate cultures are the mainstays in the clinical evaluation of suspected meningitis.

VinCENT A SPAGNA RICHARD B PRIOR

Division of Infectious Diseases, Ohio State University College of Medicine,
Columbus, Ohio 43210

Edwards, E A, Muehl, P M, and Peckinpaugh, R O fournal of Laboratory and Clinical Medicine, 1972, $80,449$.

Levin, J, and Bang, F B, Bulletin of the fohns Hopkins Hospital, 1964, 115, 265.

Jorgensen, $\mathrm{J} \mathrm{H}$, and Lee, J C, fournal of Clinical Nachum, R, Lipsey, A, and Siegel, S E, New England Journal of Medicine, 1973, 289, 931 .

Ross, S, et al, Journal of the American Medical Association, 1975, 233, 1366 .

\section{Benign presentation of tuberculous meningitis}

SIR,-We were interested in the recent controversy about benign tuberculous meningitis in your journal (6 January, p 56) and would like to describe our experience with a similar case.

In October 1970 we saw a 23-year-old Dutch nurse for. a routine physical examination. She had no symptoms, and the clinical examination was normal, as were the results of laboratory tests, including differential blood count. The Mantoux test was negative at a dilution of $1 / 10000$ but positive at $1 / 5000$. Thirteen months later she returned complaining of malaise, dizziness, syncope, recurrent pulsatile headaches, insomnia, increased irritability, and clumsiness of her right hand. The physical examination was essentially unchanged, except for a highly labile blood pressure with frequently raised values (up to $160 / 115 \mathrm{~mm} \mathrm{Hg}$ ). There were no neurological signs of lateralisation or a focal lesion. The lumbar puncture showed a pressure of $13 \mathrm{~cm} \mathrm{H}_{2} \mathrm{O}$, protein $0.20 \mathrm{~g} / \mathrm{l}$, glucose $3.39 \mathrm{mmol} / 1(0.61 \mathrm{~g} / \mathrm{l})$, a slightly positive Pandy reaction, and $1 \times 10^{6}$ mononucleated cell $/ 1$. Surprisingly, the cerebrospinal fluid (CSF) culture was positive for Mycobacterium tuberculosis, typus humanus, which was subsequently shown to be sensitive to all antibiotics tested. We wondered whether this isolated CSF finding represented an actual infection or an extrinsic contamination. A second lumbar puncture was performed, and the CSF was sterile. Her Mantoux test was now positive at 1/10000. Clinically, headache and blood pressure lability persisted for a few weeks, then progressively disappeared. Because of these facts the diagnosis of tuberculous meningitis was finally rejected and the patient discharged without specific treatment. A follow-up was not possible after six months, because the patient returned to Holland.

In view of the recent debate in your journal, this case might be another example of benign tuberculous meningitis, as described by Emond and McKendrick.' The symptoms, suggestive of raised intracranial pressure, and 